\title{
Interval Intuitionistic Trapezoidal Fuzzy Prioritized Aggregating Operators and their Application to Multiple Attribute Decision Making
}

\author{
Xian-Ping Jiang \\ Chongqing University of Arts and Sciences \\ China \\ cqmanagement@163.com
}

\begin{abstract}
In this paper, we investigate the interval intuitionistic trapezoidal fuzzy multiple attribute decision making $(M A D M)$ problems in which the attributes are in different priority level. Motivated by the ideal of prioritized aggregation operators bu R.R. Yager, we developed some prioritized aggregation operators for aggregating interval intuitionistic trapezoidal fuzzy information, and then apply them to develop some models for interval intuitionistic trapezoidal fuzzy multiple attribute decision making (MADM) problems in which the attributes are in different priority level. Finally, a practical example about talent introduction is given to verify the developed approaches and to demonstrate its practicality and effectiveness.
\end{abstract}

Keywords: Multiple Attribute Decision Making (MADM), Interval Intuitionistic Trapezoidal Fuzzy Information, Prioritized Aggregation Operators, Interval Intuitionistic Trapezoidal Fuzzy Prioritized Weighted Average (IITFPWA) Operator, Interval Intuitionistic Trapezoidal Fuzzy Prioritized Weighted Geometric (IITFPWG) Operator

Received: 2 April 2018, Revised 29April 2018, Accepted 8 May 2018

\section{DOI:}

(C) 2018 DLINE. All Rights Reserved

\section{Introduction}

Atanassov[1-3]introduced the concept of intuitionistic fuzzy set(IFS), which is a generalization of the concept of fuzzy set[4]. Atanassov and Gargov[5] introduced the concept of interval-valued intuitionistic fuzzy sets (IVIFSs) as a further generalization of that of IFSs, as well as of IVFSs. Atanassov[6] defined some operational laws of the IVIFSs. The intuitionistic fuzzy set and interval-valued intuitionistic fuzzy sets has received more and more attention since its appearance[7-26]. Shu, Cheng and Chang[27] gave the definition and operational laws of intuitionistic triangular fuzzy number and proposed an algorithm of the intuitionistic fuzzy fault-tree analysis. Wang[28] gave the definition of intuitionistic trapezoidal fuzzy number and interval intuitionistic trapezoidal fuzzy number. Wang and Zhang [29] gave the definition of expected values of intuitionistic trapezoidal fuzzy number and proposed the programming method of multi-criteria decision-making based on intuitionistic trapezoidal fuzzy number with incomplete certain information. Wang and Zhang[30] developed the Hamming distance of intuitionistic trapezoidal fuzzy numbers and intuitionistic trapezoidal fuzzy weighted arithmetic averaging (ITFWAA) operator, then proposed multi-criteria decision-making

\begin{tabular}{llllll}
\hline 102 & Journal of Intelligent Computing Volume 9 & Number 3 & September 2018 \\
\hline
\end{tabular}


method with incomplete certain information based on intuitionistic trapezoidal fuzzy number. Wan[31] defined the score function and accuracy function of an interval intuitionistic trapezoidal fuzzy number and proposed the interval intuitionistic trapezoidal fuzzy weighted average (IITFWA) operator and the interval intuitionistic trapezoidal fuzzy weighted geometric(IITFWG) operator. Wei[32] investigated the multiple attribute group decision making (MAGDM) problems in which both the attribute weights and the expert weights takes the form of real numbers, attribute values takes the form of interval intuitionistic trapezoidal fuzzy numbers. Then some new aggregation operators including interval intuitionistic trapezoidal fuzzy ordered weighted geometric (IITFOWG) operator and interval intuitionistic trapezoidal fuzzy hybrid geometric (IITFHG) operator, are proposed and some desirable properties of these operators are studied, such as commutativity, idempotency and monotonicity. Then, an IITFWG and IITFHG operators-based approach is developed to solve the MAGDM problems in which both the attribute weights and the expert weights take the form of real numbers, attribute values take the form of interval intuitionistic trapezoidal fuzzy numbers. Finally, some illustrative examples are given to verify the developed approach and to demonstrate its practicality and effectiveness.

From above analysis, we can see that interval intuitionistic trapezoidal fuzzy set is a very useful tool to deal with uncertainty. More and more multiple attribute decision making theories and methods under interval intuitionistic trapezoidal fuzzy environment have been developed. Current methods are under the assumption that the attributes are at the same priority level. However, in real and practical multiple attribute decision making problem, the attributes have different priority level commonly. To overcome this drawback, motivated by the ideal of prioritized aggregation operators [33-34], in this paper, we propose some interval intuitionistic trapezoidal fuzzy prioritized aggregation operators: interval intuitionistic trapezoidal fuzzy prioritized weighted average (IITFPWA) operator and interval intuitionistic trapezoidal fuzzy prioritized weighted geometric (IITFPWG) operator. The prominent characteristic of these proposed operators is that they take into account prioritization among the attributes. Then, we have utilized these operators to develop some approaches to solve the interval intuitionistic trapezoidal fuzzy multiple attribute decision making problems in which the attributes are in different priority level. To do so, the remainder of this paper is set out as follows. In the next section, we introduce some basic concepts related to interval intuitionistic trapezoidal fuzzy numbers and some operational laws of interval intuitionistic trapezoidal fuzzy numbers. In Section 3 we have developed some interval intuitionistic trapezoidal fuzzy prioritized aggregation operators: interval intuitionistic trapezoidal fuzzy prioritized weighted average (IITFPWA) operator and interval intuitionistic trapezoidal fuzzy prioritized weighted geometric (ITTFPWG) operator and studied some desirable properties of the proposed operator. The prominent characteristic of these proposed operators is that they take into account prioritization among the attributes. In Section 4, we have developed apply these operators to develop some models for interval intuitionistic trapezoidal fuzzy multiple attribute decision making (MADM) problems in which the attributes are in different priority level. In Section 5, a practical example about talent introduction is given to verify the developed approach and to demonstrate its practicality and effectiveness. In Section 6, we conclude the paper and give some remarks.

\section{Preliminaries}

\subsection{Interval Intuitionistic Trapezoidal Fuzzy Numbers Set}

In the following, we shall introduce some basic concepts related to intuitionistic trapezoidal fuzzy numbers and interval intuitionistic trapezoidal fuzzy numbers.

Definition 1[28]. Let $\tilde{a}$ is an intuitionistic trapezoidal fuzzy number, its membership function is:

$$
\mu_{\tilde{a}}(x)=\left\{\begin{array}{l}
\frac{x-a}{b-a} \mu_{\tilde{a}}, a \leq x<b ; \\
\mu_{\tilde{a}}, b \leq x \leq c ; \\
\frac{d-x}{d-c} \mu_{\tilde{a}}, c<x \leq d ; \\
0, \text { others }
\end{array}\right.
$$

Its Non-membership function is: 


$$
v_{\tilde{a}}(x)=\left\{\begin{array}{l}
\frac{b-x+v_{\tilde{a}}\left(x-a_{1}\right)}{b-a_{1}}, a_{1} \leq x<b ; \\
\frac{v_{\tilde{a}}, b \leq x \leq c ;}{d_{1}-c+v_{\tilde{a}}\left(d_{1}-x\right)} \\
0, c<x \leq d_{1} ; \\
\text { others. }
\end{array}\right.
$$

Where $0 \leq \mu_{\tilde{a}} \leq 1 ; 0 \leq v_{\tilde{a}} \leq 1 ;$ and $\mu_{\tilde{a}}+v_{\tilde{a}} \leq 1 ; a, b, c, d \in R$. Then $\tilde{a}=<\left([a, b, c, d] ; \mu_{\tilde{a}}\right),\left(\left[a_{1}, b, c, d_{1}\right] ; v_{\tilde{a}}\right)>$ is called an intuitionistic trapezoidal fuzzy number. For convenience, let $\tilde{a}=\left([a, b, c, d] ; \mu_{\tilde{a}}, v_{\tilde{a}}\right)$.

If $\tilde{\mu}_{\mathrm{A}}(x) \subset[0,1]$ and $\tilde{v}_{\mathrm{A}}(x) \subset[0,1]$ are interval numbers, and $0 \leq \sup \left(\tilde{\mu}_{\mathrm{A}}(x)\right)+\sup \left(\tilde{v}_{\mathrm{A}}(x)\right) \leq 1, \forall x \in X$, for convenience, let $\tilde{\mu}_{\mathrm{A}}$ $(x)=[\underline{\mu}, \bar{\mu}], \tilde{v}_{\mathrm{A}}(x)=[\underline{v}, \bar{v}]$, Then $\tilde{a}=\left([a, b, c, d] ; \tilde{\mu}_{\tilde{a}}, \tilde{v}_{\tilde{a}}\right)=([a, b, c, d] ;[\underline{\mu}, \bar{\mu}],[\underline{v}, \bar{v}])$ is called an interval intuitionistic trapezoidal fuzzy number[31].

Definition 2[31]. Let $\tilde{a}_{1}=\left(\left[a_{1}, b_{1}, c_{1}, d_{1}\right] ;\left[\underline{\mu_{1}}, \bar{\mu}_{1}\right],\left[\underline{\nu_{1}}, \bar{v}_{1}\right]\right)$ and $\tilde{a}_{2}=\left(\left[a_{2}, b_{2}, c_{2}, d_{2}\right] ;\left[\underline{\mu}_{2}, \bar{\mu}_{2}\right],\left[\underline{v}_{2}, \bar{v}_{2}\right]\right)$ be two interval intuitionistic trapezoidal fuzzy number, and $\lambda \geq 0$, then

$$
\begin{aligned}
& \text { (1) } \tilde{a}_{1} \tilde{a}_{2}=\left(\left[a_{1} a_{2}, b_{1} b_{2}, c_{1} c_{2}, d_{1} d_{2}\right]\right) ; \\
& \left.\left[\underline{\mu}_{1} \cdot \underline{\mu}_{2}, \bar{\mu}_{1} \cdot \bar{\mu}_{2}\right],\left[\underline{v}_{1}+\underline{v}_{2}-\underline{v}_{1} \cdot \underline{v}_{2}, \bar{v}_{1}+\bar{v}_{2}-\bar{v}_{1} \cdot \bar{v}_{2}\right]\right) ; \\
& \text { (2) } \tilde{a}_{1}^{\lambda}=\left(\left[a_{1}^{\lambda}, b_{1}^{\lambda}, c_{1}^{\lambda}, d_{1}^{\lambda}\right] ;\left[\underline{\mu}_{1}^{\lambda}, \bar{\mu}_{1}^{\lambda}\right],\left[1-\left(1-\underline{v}_{1}\right)^{\lambda}, 1-\left(1-\bar{v}_{1}\right)^{\lambda}\right]\right) .
\end{aligned}
$$

Definition 3. Let $\tilde{a}=([a, b, c, d] ;[\underline{\mu}, \bar{\mu}],[\underline{v}, \bar{v}])$ be an interval intuitionistic trapezoidal fuzzy number, a score function $S$ of an interval intuitionistic trapezoidal fuzzy number can be represented as follows:

$$
S\left(\tilde{a}_{1}\right)=\frac{a+b+c+d}{4} \cdot \frac{2+\underline{\mu}-\underline{v}+\bar{\mu}-\bar{v}}{4}, S(\tilde{a}) \in[-1,1] .
$$

Definition 4[31]. Let $\tilde{a}=([a, b, c, d] ;[\underline{\mu}, \bar{\mu}],[\underline{\underline{v}}, \bar{v}])$ be an interval intuitionistic trapezoidal fuzzy number, an accuracy function $H$ of an interval intuitionistic trapezoidal fuzzy number can be represented as follows:

$$
H(\tilde{a})=\frac{a+b+c+d}{4} \bullet \frac{\underline{\mu}+\underline{v}+\bar{\mu}+\bar{v}}{2}, H(\tilde{a}) \in[0,1] .
$$

to evaluate the degree of accuracy of the interval intuitionistic trapezoidal fuzzy number $\tilde{a}$, where $H(\tilde{a}) \in[0,1]$. The larger the value of $H(\tilde{a})$, the more the degree of accuracy of the interval intuitionistic trapezoidal fuzzy number $\tilde{a}$.

As presented above, the score function $S$ and the accuracy function $H$ are, respectively, defined as the difference and the sum of the membership function $\tilde{\mu}_{\mathrm{A}}(\mathrm{x})$ and the non-membership function $\tilde{v}_{\mathrm{A}}(\mathrm{x})$. Based on the score function $S$ and the accuracy function $H$, in the following, Wan[31] give an order relation between two interval intuitionistic trapezoidal fuzzy number, which is defined a follows:

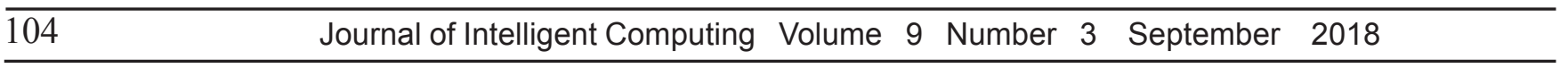


Definition 5. Let $\tilde{a}_{1}=\left(\left[a_{1}, b_{1}, c_{1}, d_{1}\right] ;\left[\underline{\mu}_{1}, \bar{\mu}_{1}\right],\left[\underline{\nu}_{1}, \bar{v}_{1}\right]\right)$ and $\tilde{a}_{2}=\left(\left[a_{2}, b_{2}, c_{2}, d_{2}\right] ;\left[\underline{\mu}_{2}, \bar{\mu}_{2}\right],\left[\underline{\nu}_{2}, \bar{v}_{2}\right]\right)$ be two interval intuitionistic trapezoidal fuzzy number, $s\left(\tilde{a}_{1}\right)$ and $s\left(\tilde{a}_{2}\right)$ be the scores of $\tilde{a}$ and $\widetilde{b}$, respectively, and let $H\left(\tilde{a}_{1}\right)$ and $H\left(\tilde{a}_{2}\right)$ be the accuracy degrees of $\tilde{a}$ and $\widetilde{b}$, respectively, then if $S(\tilde{a})<S(\widetilde{b})$, then $\tilde{a}$ is smaller than $\widetilde{b}$, denoted by $\tilde{a}<\widetilde{b} ;$ if $S(\tilde{a})<S(\widetilde{b})$, then

if $H(\tilde{a})=H(\tilde{b})$, then $\tilde{a}$ and $\tilde{b}$ represent the same information, denoted by $\tilde{a}=\tilde{b} ;(2)$ if $H(\tilde{a})=H(\tilde{b}), \tilde{a}$ is smaller than $\tilde{b}$, denoted by $\tilde{a}<\tilde{\mathrm{b}}[10-11]$.

\subsection{Prioritized Average (PA) Operator}

The prioritized average (PA) operator was originally introduced by Yager [33], which was defined as follows:

Definition 6[33]. Let $G=\left\{G_{1}, G_{2}, \ldots, G_{n}\right\}$ be a collection of attribute and that there is a prioritization between the attribute expressed by the linear ordering $G_{1} \succ G_{2} \succ G_{3} \ldots . \succ G_{n}$, indicate attribute $G_{j}$ has a higher priority than $G_{k}$, if $j<k$. The value $G_{j}(x)$ is the performance of any alternative $x$ under attribute $G_{j}$, and satisfies $G_{j}(x) \in[0,1]$. If

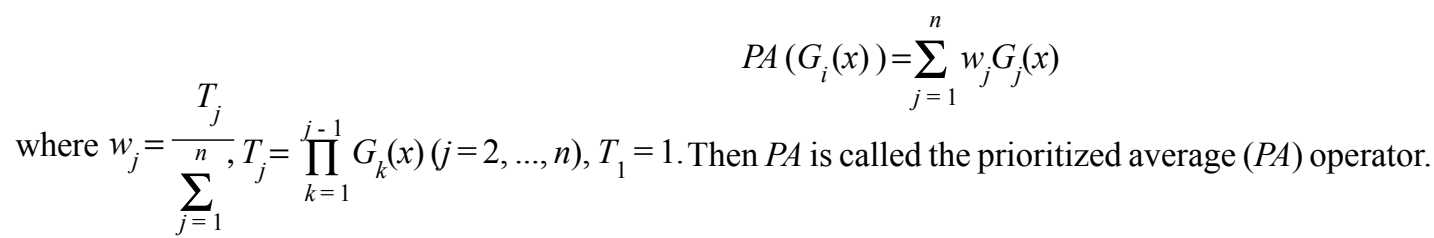

\section{Interval Intuitionistic Trapezoidal Fuzzy Prioritized Aggregation Operators}

\subsection{Interval Intuitionistic Trapezoidal Fuzzy Prioritized Weighted Average (IITFPWA) Operator}

The prioritized average [33] operators, however, have usually been used in situations where the input arguments are the exact values. We shall extend the PA operators to accommodate the situations where the input arguments are interval intuitionistic trapezoidal fuzzy information. In this Section, we shall investigate the PA operator under interval intuitionistic trapezoidal fuzzy environments. Based on Definition 6, we give the definition of the interval intuitionistic trapezoidal fuzzy prioritized weighted average (IITFPWA) operator as follows:

Definition 7. Let $\tilde{a}_{j}(j=1,2, \ldots, n)$ be a collection of interval intuitionistic trapezoidal fuzzy numbers, then we define the interval intuitionistic trapezoidal fuzzy prioritized weighted average (IITFPWA) operator as follows:

$$
\begin{gathered}
\operatorname{IITFPWA}\left(\tilde{a}_{1}, \tilde{a}_{2}, \ldots, \tilde{a}_{n}\right) \\
=\frac{T_{1}}{\sum_{j=1}^{n} T_{j}} \tilde{a}_{1} \oplus \frac{T_{2}}{\sum_{j=1}^{n} T_{j}} \tilde{a}_{2} \oplus \ldots \oplus \frac{T_{n}}{\sum_{j=1}^{n} T_{j}} \tilde{a}_{n}=\stackrel{\oplus}{j=1}^{n}\left(\frac{T_{j} \tilde{a}_{j}}{\sum_{j=1}^{n} T_{j}}\right)
\end{gathered}
$$

where $T_{j}=\prod_{k=1}^{j-1} s\left(\tilde{a}_{j}\right)(j=2, \cdots, n), T_{1}=1$ and $s\left(\tilde{a}_{j}\right)$ is the score values of $\tilde{a}_{j}(j=1,2, \ldots, n)$.

Based on operations of the interval intuitionistic trapezoidal fuzzy information described in Section 2.1, We can drive the Theorem 1.

Theorem 1. Let $\tilde{a}_{j}(j=1,2, \ldots, n)$ be a collection of interval intuitionistic trapezoidal fuzzy numbers, then their aggregated value by using the IITPWA operator is also an interval ntuitionistic trapezoidal fuzzy number, and

$$
\begin{gathered}
\operatorname{IITFPWA}\left(\tilde{a}_{1}, \tilde{a}_{2}, \ldots, \tilde{a}_{n}\right) \\
=\frac{T_{1}}{\sum_{j=1}^{n} T_{j}} \tilde{a}_{1} \oplus \frac{T_{2}}{\sum_{j=1}^{n} T_{j}} \tilde{a}_{2} \oplus \ldots \oplus \frac{T_{n}}{\sum_{j=1}^{n} T_{j}} \tilde{a}_{n}=\stackrel{\oplus}{j=1}^{n}\left(\frac{T_{j} \tilde{a}_{j}}{\sum_{j=1}^{n} T_{j}}\right)
\end{gathered}
$$




$$
\begin{gathered}
=\left(\left[\sum_{j=1}^{n} \frac{T_{j} a_{j}}{\sum_{j=1}^{n} T_{j}}, \sum_{j=1}^{n} \frac{T_{j} b_{j}}{\sum_{j=1}^{n} T_{j}}, \sum_{j=1}^{n} \frac{T_{j} c_{j}}{\sum_{j=1}^{n} T_{j}}, \sum_{j=1}^{n} \frac{T_{j} d_{j}}{\sum_{j=1}^{n} T_{j}}\right] ;\right. \\
\left.\left[1-\prod_{j=1}^{n}\left(1-\underline{\mu}_{j}\right) \frac{T_{j}}{\sum_{j=1}^{n} T_{j}}, 1-\prod_{j=1}^{n}\left(1-\bar{\mu}_{j}\right) \frac{T_{j}}{\sum_{j=1}^{n} T_{j}}\right],\left[\begin{array}{c}
\frac{T_{j}}{\sum_{j=1}^{n} T_{j}} \frac{T_{j}}{\sum_{j=1}^{n} T_{j}} \\
\prod_{j=1}^{n} \underline{v}_{j}^{n} \bar{v}_{j}
\end{array}\right]\right)
\end{gathered}
$$

where $T_{j}=\prod_{k=1}^{j-1} s\left(\tilde{a}_{j}\right)(j=2, \ldots, n), T_{1}=1$ and $s(\tilde{a} j)$ is the score values of $\tilde{a}_{j}(j=2, \ldots, n)$.

It can be easily proved that the IITFPWA operator has the following properties.

Theorem 2. (Idempotency) Let $\tilde{a}_{j}(j=1,2, \ldots, n)$ be a collection of interval intuitionistic trapezoidal fuzzy numbers, where $T_{j}=\prod_{k=1}^{j-1} s\left(\tilde{a}_{j}\right)(j=2, \ldots, n), T_{1}=1$ and $s\left(\tilde{a}_{j}\right)$ is the score values of $\tilde{a}_{i}(j=1,2, \ldots, n)$. If all $\tilde{a}_{j}(j=1,2, \ldots, n)$ are equal, i.e. $\tilde{a}_{j}=\tilde{a}$ for all $j$, then

$$
\operatorname{IITFPWA}\left(\tilde{a}_{1}, \tilde{a}_{2}, \ldots, \tilde{a}_{n}\right)=\tilde{a}
$$

Theorem 3. (Boundedness) Let $\tilde{a}_{j}(j=1,2, \ldots, n)$ be a collection of interval intuitionistic trapezoidal fuzzy numbers, where $T_{j}=\prod_{k=1}^{j-1} s\left(\tilde{a}_{j}\right)(j=2, \ldots, n), T_{1}=1$ and $s\left(\tilde{a}_{j}\right)$ is the score values of $\tilde{a}_{j}(j=1,2, \ldots, n)$., and let

Then

$$
\tilde{a}^{-}=\min _{j} \tilde{a}_{j}, \tilde{a}^{+}=\max _{j} \tilde{a}_{j}
$$

$$
\tilde{a}^{-} \leq \operatorname{IITFPWA}\left(\tilde{a}_{1}, \tilde{a}_{2}, \ldots, \tilde{a}_{n}\right) \leq \tilde{a}^{+}
$$

Theorem 4. (Monotonicity) Let $\tilde{a}_{j}(j=1,2, \ldots, n)$ and $\tilde{a}_{j}^{\prime}(j=1,2, \ldots, n)$ be two set of interval intuitionistic trapezoidal fuzzy numbers, where $T_{j}=\prod_{k=1}^{j-1} s\left(\tilde{a}_{j}\right) T_{j}^{\prime}=\prod_{k=1}^{j-1} s\left(\tilde{a}_{j}^{\prime}\right)(j=1,2, \ldots, n), T_{1}=T_{1}^{\prime}=1, s\left(\tilde{a}_{j}\right)$ is the is the score values of $\tilde{a}_{j}(j=1,2, \ldots, n), s\left(\tilde{a}_{j}^{\prime}\right)$ is the score values of $\tilde{a}_{j}^{\prime}(j=1,2, \ldots, n)$, if $\tilde{a}_{j} \leq \tilde{a}_{j}^{\prime}$, for all $j$,

then

$$
\operatorname{IITFPWA}\left(\tilde{a}_{1}, \tilde{a}_{2}, \ldots, \tilde{a}_{n}\right) \leq \operatorname{IITFPWA}\left(\tilde{a}_{1}^{\prime}, \tilde{a}_{2}^{\prime}, \ldots, \tilde{a}_{n}^{\prime}\right)
$$

\subsection{Interval Intuitionistic Trapezoidal Fuzzy Prioritized Weighted Geometric (IITFPWG) Operator}

Based on the IITFPWA operator and the geometric mean, here we define an interval intuitionistic trapezoidal fuzzy prioritized weighted geometric (IITFPWG) operator:

Definition 8. Let $\tilde{a}_{j}(j=1,2, \ldots, n)$ be a collection of interval intuitionistic trapezoidal fuzzy numbers, then we define the interval intuitionistic trapezoidal fuzzy prioritized weighted geometric (IITFPWG) operator as follows:

$$
\operatorname{IITFPWG}\left(\tilde{a}_{1}, \tilde{a}_{2}, \ldots, \tilde{a}_{n}\right)
$$




$$
=\frac{T_{1}}{\sum_{1}^{n}} T_{j=1} \frac{T_{2}}{\sum_{j=1}^{n}} T_{j} \otimes \ldots \otimes \tilde{a}_{n}^{\sum_{j=1}^{n}} T_{j}=\bigotimes_{j=1}^{n}\left(\sum_{\tilde{a}_{j}^{j=1}}^{\frac{T_{j}}{\sum^{n}}} T\right)
$$

where $T_{j}=\prod_{k=1}^{j-1} s\left(\tilde{a}_{j}\right)(j=2, \ldots, n), T_{1}=1$ and $s\left(\tilde{a}_{j}\right)$ is the score values of $\tilde{a}_{j}(j=1,2, \ldots, n)$.

Based on operations of the interval intuitionistic trapezoidal fuzzy numbers described in Section 2, we can drive the Theorem 5.

Theorem 5. Let $\tilde{a}_{j}(j=1,2, \ldots, n)$ be a collection of interval intuitionistic trapezoidal fuzzy numbers, then their aggregated value by using the IITPWA operator is also an interval intuitionistic trapezoidal fuzzy number, and

$$
\begin{aligned}
& \operatorname{IITFPWG}\left(\tilde{a}_{1}, \tilde{a}_{2}, \ldots, \tilde{a}_{n}\right) \\
& =\tilde{a}_{1}^{\frac{T_{1}}{\sum_{j=1}^{n}} T_{j} \otimes \tilde{a}_{2}^{\sum_{j=1}^{n}}} T_{j} \otimes \ldots \otimes \tilde{a}_{n}^{\frac{T_{2}}{\sum_{j=1}^{n}}} T_{j}=\otimes_{j=1}^{n}\left(\frac{T_{j}}{\sum_{\tilde{a}_{j}^{j=1}}^{n} T_{j}}\right)
\end{aligned}
$$

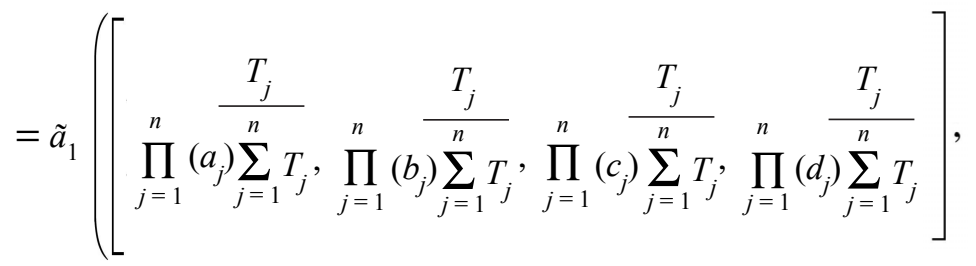

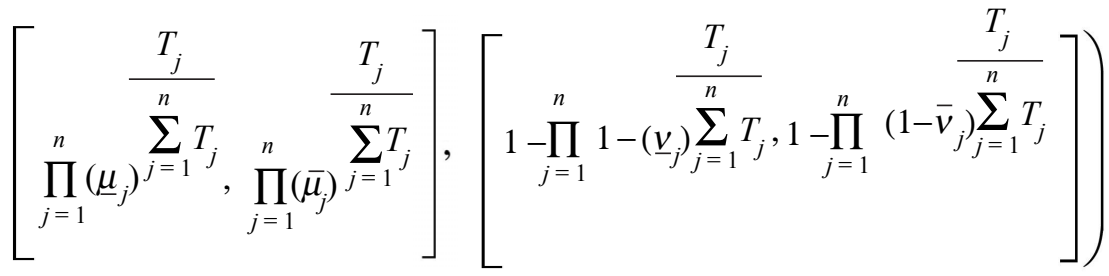

where $T_{j}=\prod_{k=1}^{j-1} s\left(\tilde{a}_{j}\right)(j=2, \ldots, n), T_{1}=1$ and $s\left(\tilde{a}_{j}\right)$ is the score values of $\tilde{a}_{j}(j=1,2, \ldots, n)$.

It can be easily proved that the IITFPWG operator has the following properties.

Theorem 6. (Idempotency) Let $\tilde{a}_{j}(j=1,2, \cdots, n)$ be a collection of interval intuitionistic trapezoidal fuzzy numbers, where $T_{j}=\prod_{k=1}^{j-1} s\left(\tilde{a}_{j}\right)(j=2, \cdots, n), T_{1}=1$ and $s\left(\tilde{a}_{j}\right)$ is the score values of $\tilde{a}_{j}(j=1,2, \cdots, n)$. If all $\tilde{a}_{j}(j=1,2, \cdots, n)$ are equal, i.e. $\tilde{a}_{j}=\tilde{a}$ for all $j$, then

$$
\operatorname{IITFPWG}\left(\tilde{a}_{1}, \tilde{a}_{2}, \cdots, \tilde{a}_{n}\right)=\tilde{a}
$$

Theorem 7. (Boundedness) Let $\left(\tilde{a}_{j}\right)(j=1,2, \ldots, n)$ be a collection of interval intuitionistic trapezoidal fuzzy numbers, where (13) 
$T_{j}=\prod_{k=1}^{j-1} s\left(\tilde{a}_{j}\right)(j=2, \ldots, n), T_{1}=1$ and $s\left(\tilde{a}_{j}\right)$ is the score values of $\tilde{a}_{j}(j=1,2, \ldots, n)$ and let

then

$$
\tilde{a}^{-}=\min _{j} \tilde{a}_{j}, \tilde{a}^{+}=\max _{j} \tilde{a}_{j}
$$

$$
\tilde{a}^{-} \leq \operatorname{IITFPWG}\left(\tilde{a}_{1}, \tilde{a}_{2}, \ldots, \tilde{a}_{n}\right) \leq \tilde{a}^{+}
$$

Theorem 8. (Monotonicity) Let $\tilde{a}_{j}(j=1,2, \ldots, n)$ and $\tilde{a}_{j}{ }_{j}(j=1,2, \ldots, n)$ be two set of interval intuitionistic trapezoidal fuzzy numbers, where $T_{j}=\prod_{k=1}^{j-1} s_{j}\left(\tilde{a}_{j}\right), T_{j}=\prod_{k=1}^{j-1} s_{j}\left(\tilde{a}_{j}^{\prime}\right)(j=1,2, \ldots, n), T_{1}=T_{1}^{\prime}=1, s\left(\tilde{a}_{j}\right)$ is the score values of $\tilde{a}_{j}^{\prime}(j=1,2, \ldots, n)$ if $\tilde{a}_{j}<\tilde{a}_{j}{ }_{j}$ for all $j$, then

$$
\operatorname{IITFPWG}\left(\tilde{a}_{1}, \tilde{a}_{2}, \ldots, \tilde{a}_{n}\right) \leq \operatorname{IITFPWA}\left(\tilde{a}_{1}^{\prime}, \tilde{a}_{2}^{\prime}, \ldots, \tilde{a}_{n}^{\prime}\right)
$$

\section{An Approach to Multiple Attribute Decision Making with Interval Intuitionistic Trapezoidal Fuzzy Information}

In this section, we shall utilize the interval intuitionistic trapezoidal prioritized aggregation operators to multiple attribute decision making with interval intuitionistic trapezoidal fuzzy information.

For a multiple attribute decision making problems with interval intuitionistic trapezoidal fuzzy information, let $X=\left\{X_{1}, X_{2}, \ldots, X_{m}\right\}$ be a discrete set of alternatives, Let $G=\left\{G_{1}, G_{2}, \ldots, G_{n}\right\}$ be a collection of attribute and that there is a prioritization between the attribute expressed by the linear ordering $G_{1} \succ G_{2} \succ G_{3} \ldots \succ G_{n}$, indicate attribute $G_{j}$ has a higher priority than $G_{s}$. If $j<s$ the decision makers provide several values for the alternative $A_{i}$ under the attribute $G_{j}$ with anonymity, these values can be considered as an interval intuitionistic trapezoidal $\tilde{h}_{i j}$. In the case where two decision makers provide the same value, then the value emerges only once in $\tilde{h}_{i j}$. Suppose that $\tilde{H}=\left(\tilde{h}_{i j}\right)_{m \times n}=\left(\left[a_{i j}, b_{i j}, c_{i j}, d_{i j}\right] ; \tilde{\mu}_{i j}, \tilde{v}_{i j}\right)_{m \times n}$ is the interval intuitionistic trapezoidal fuzzy decision matrix, where $\tilde{\mu}_{\mathrm{ij}}$ indicates the degree that the alternative $A_{i}$ satisfies the attribute $G_{j}$ given by the decision maker, $\tilde{v}_{\mathrm{ij}}$ indicates the degree that the alternative $A_{i}$ doesn't satisfy the attribute $G_{j}$ given by the decision maker, $\tilde{\mu}_{\mathrm{ij}} \subseteq[0,1], \quad \tilde{v}_{\mathrm{ij}} \subseteq[0,1], \quad \bar{\mu}_{\mathrm{ij}}+\bar{v}_{\mathrm{ij}} \leq 1, i=1,2, \ldots m, j=1,2, \ldots n$.

Then, we utilize the IITFPWA (or IITFPWG) operator to develop an approach to multiple attribute decision making problems with interval intuitionistic trapezoidal fuzzy information, which can be described as following:

Step 1. Calculate the values of $T_{i j}(i=1,2, \ldots m, j=1,2, \ldots n)$ as follows

$$
\begin{gathered}
T_{i j}=\prod_{\lambda=1}^{j-1} s\left(\tilde{h}_{i j}\right),(1,2, \ldots m, j=1,2, \ldots n) \\
T_{i 1}=1, i=1,2, \ldots m
\end{gathered}
$$

Step 2. Aggregate all interval intuitionistic trapezoidal fuzzy numbers $\widetilde{h}_{i j}(j=1,2, \ldots n)$ by using the interval intuitionistic trapezoidal fuzzy prioritized weighted average (IITFPWA) operator:

$$
\begin{gathered}
\tilde{h}_{i}=\operatorname{IITFPWA}\left(\tilde{h}_{i 1}, \tilde{h}_{i 2}, \cdots, \tilde{h}_{i n}\right) \\
=\frac{T_{i 1}}{\sum_{j=1}^{n} T_{i j}} \tilde{h}_{i 1} \oplus \frac{T_{i 2}}{\sum_{j=1}^{n} T_{i j}} \tilde{h}_{i 2} \oplus \cdots \oplus \frac{T_{i n}}{\sum_{j=1}^{n} T_{i j}} \tilde{h}_{i n}=\bigoplus_{j=1}^{n}\left(\frac{T_{j} \tilde{h}_{i j}}{\sum_{j=1}^{n} T_{i j}}\right)
\end{gathered}
$$




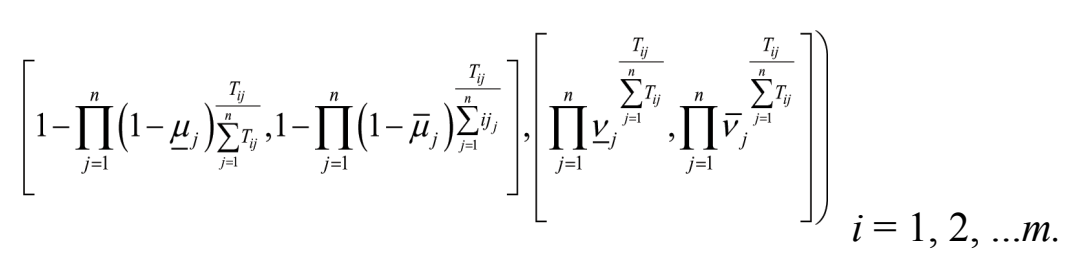

Or the interval intuitionistic trapezoidal fuzzy prioritized weighted geometric (IITFPWG) operator:

$$
\begin{aligned}
& \tilde{h}_{i}=\operatorname{IITFPWG}\left(\tilde{h}_{i 1}, \tilde{h}_{i 2}, \cdots, \tilde{h}_{i n}\right) \\
& =\tilde{h}_{i 1}^{\frac{T_{i 1}}{\sum_{j=1}^{n} T_{i j}}} \otimes \tilde{h}_{i 2} \frac{T_{i 2}}{\sum_{j=1}^{n} T_{i j}} \otimes \cdots \otimes \tilde{h}_{i n} \frac{T_{i n}}{\sum_{j=1}^{n} T_{i j}} \\
& =\bigotimes_{j=1}^{n}\left(\tilde{h}_{i j}^{\frac{T_{i j}}{\sum_{j=1}^{n} T_{i j}}}\right) \\
& =\left(\left[\prod_{j=1}^{n}\left(a_{i j}\right)^{\frac{T_{i j}}{n} T_{i j}}, \prod_{j=1}^{n}\left(b_{i j}\right)^{\frac{T_{i j}}{n} \sum_{j=1}^{n}}, \prod_{j=1}^{n}\left(c_{i j}\right)_{j=1}^{\frac{T_{i j}}{n} T_{i j}}, \prod_{j=1}^{n}\left(d_{i j}\right)^{\frac{T_{i j}}{\sum_{j=1}^{n} T_{i j}}}\right] ;\right. \\
& \left.\left[\prod_{j=1}^{n} \underline{\mu}_{i j}^{\frac{T_{i j}}{\sum_{j=1}^{n} T_{i j}}}, \prod_{j=1}^{n} \bar{\mu}_{i j}^{\frac{T_{i j}}{\sum_{j=1}^{n} T_{i j}}}\right],\left[1-\prod_{j=1}^{n}\left(1-\underline{v}_{i j}\right)_{j=1}^{\frac{T_{i j}}{n} \sum_{i j}}, 1-\prod_{j=1}^{n}\left(1-\bar{v}_{i j}\right)_{j=1}^{\frac{T_{i j}}{n \sum_{j i j} T_{i j}}}\right]\right) \\
& i=1,2, \cdots, m \text {. }
\end{aligned}
$$

Step 3. Calculate the scores $S\left(\tilde{h}_{i j}\right)(i=1,2, \ldots m)$ of the overall interval intuitionistic trapezoidal fuzzy preference values $\tilde{h}_{i}(i=1,2$, ...m) to rank all the alternatives $A_{i}(i=1,2, \ldots m)$ and then to select the best one(s).

Step 4. Rank all the alternatives $A_{i}(i=1,2, \ldots m)$ and select the best one(s) in accordance with $S\left(\widetilde{h}_{i j}\right)(i=1,2, \ldots m)$.

Step 5. End.

\section{Numerical Example}

In order to strengthen academic education, promote the building of teaching body, the school of management in a Chinese university wants to introduce oversea outstanding teachers (adapted from [35]). This introduction has been raised great attention from the school, university president, dean of management school and human resource officer sets up the panel of decision makers which will take the whole responsibility for this introduction. They made strict evaluation for 5 candidates $X_{i}(i=1,2,3,4$, 5) according to the following four attributes: (1) $G 1$ is the morality; (2) $G 2$ is the research capability; (3) $G 3$ is the teaching skill; (4) $G 4$ is the education background University president have the absolute priority for decision making, dean of the management school comes next. Besides, this introduction will be in strict accordance with the principle of combine ability with political integrity. The prioritization relationship for the criteria is as below, $G_{1} \succ G_{2} \succ G_{3} \ldots \succ G_{n}$. The five possible candidates $X_{i}(i=1$, $2,3,4,5)$ are to be evaluated using the interval intuitionistic trapezoidal fuzzy values by the three decision makers under the above four attributes, and construct the decision matrix as listed in the following matrices $\tilde{H}=\left(\tilde{h}_{i j}\right)_{5 \times 4}$ as follows: 


$$
\begin{aligned}
& \tilde{H}=\quad\left[\begin{array}{l}
{[(0.5,0.6,0.7,0.8] ;[0.4,0.5] ;[0.3,0.4] ;[0.1,0.2,0.3,0.4] ;[0.4,0.5] ;[0.1,0.2])} \\
{[(0.6,0.7,0.8,0.9] ;[0.2,0.7] ;[0.2,0.3] ;[0.5,0.6,0.7,0.8] ;[0.3,0.6] ;[0.2,0.4])} \\
{[(0.1,0.2,0.4,0.5] ;[0.3,0.4] ;[0.1,0.2] ;[0.2,0.3,0.5,0.6] ;[0.3,0.5] ;[0.1,0.4]} \\
[(0.3,0.4,0.5,0.6)] ;[0.3,0.6][0.2,0.4] ;[0.1,0.3,0.4,0.5] ;[0.4,0.6] ;[0.2,0.3]) \\
{[(0.2,0.3,0.4,0.5] ;[0.4,0.7] ;[0.1,0.3] ;[0.3,0.4,0.5,0.6] ;[0.5,0.6] ;[0.3,0.4])}
\end{array}\right. \\
& {[(0.5,0.6,0.8,0.9] ;[0.3,0.6] ;[0.2,0.3] ;[0.4,0.5,0.6,0.7] ;[0.3,0.7] ;[0.1,0.3])} \\
& {[(0.4,0.5,0.7,0.8] ;[0.4,0.7] ;[0.1,0.2] ;[0.5,0.6,0.7,0.9] ;[0.5,0.8] ;[0.1,0.2])} \\
& [0.5,0.6,0.7,0.8] ;[0.2,0.6] ;[0.2,0.3] ;[0.3,0.5,0.7,0.9] ;[0.2,0.4] ;[0.1,0.5]) \\
& [0.1,0.3,0.5,0.7] ;[0.3,0.6] ;[0.1,0.4] ;[0.6,0.7,0.8,0.9] ;[0.3,0.7] ;[0.1,0.2]) \\
& [0.2,0.3,0.4,0.5] ;[0.2,0.5] ;[0.3,0.4] ;[0.5,0.6,0.7,0.8] ;[0.5,0.6] ;[0.2,0.4])
\end{aligned}
$$

Then, in order to select the most desirable candidate, we utilize the IITFPWA operator to develop an approach to multiple attribute decision making problems with interval intuitionistic trapezoidal fuzzy information, which can be described as following:

Step 1. Utilize (19)-(20) to calculate the values of $T_{i j}(i=1,2, \ldots m, j=2, \ldots n)$ as follows:

$$
T_{i j}=\left[\begin{array}{llll}
1.0000 & 0.3575 & 0.0581 & 0.0244 \\
1.0000 & 0.4500 & 0.1682 & 0.0706 \\
1.0000 & 0.1800 & 0.0414 & 0.0155 \\
1.0000 & 0.2588 & 0.0526 & 0.0126 \\
1.0000 & 0.2363 & 0.0638 & 0.0112
\end{array}\right]
$$

\begin{tabular}{|c|c|c|c|}
\hline$\overline{11}$ & Journal of Intelligent Computing & Volume $s$ & 9 Number \\
\hline
\end{tabular}

Step 2. Aggregate all interval intuitionistic trapezoidal fuzzy numbers by $\tilde{h}_{\mathrm{ij}}(\mathrm{j}=1,2, \cdots, n)$ using the interval intuitionistic trapezoidal fuzzy prioritized weighted average (IITFPWA) operator to derive the overall interval intuitionistic trapezoidal fuzzy numbers $\tilde{\mathrm{h}}_{i}(\mathrm{i}=1,2, \cdots, 5)$ of the candidates $A_{i}$.

$$
\begin{aligned}
& ([0.3990,0.4990,0.6030,0.7030] ;[0.5882,0.4975] ;[0.2268,0.3259]) \\
& ([0.5493,0.6493,0.7592,0.8634] ;[0.3937,0.6588] ;[0.2023,0.2855]) \\
& ([0.1304,0.2317,0.4283,0.5296] ;[0.3790,0.4101] ;[0.1047,0.2238]) \\
& ([0.2558,0.3793,0.4833,0.5873] ;[0.4555,0.5921] ;[0.2042,0.3555]) \\
& ([0.2206,0.3206,0.4206,0.5206] ;[0.4961,0.6678] ;[0.1326,0.3167])
\end{aligned}
$$

Step 3. Calculate the scores $\tilde{h}_{i}(i=1,2, \cdots, 5)$ of the overall interval intuitionistic trapezoidal fuzzy numbers $\tilde{h}_{i}(i=1,2, \cdots, 5)$ of the candidates $A_{i}$ : 


$$
\begin{aligned}
& S\left(\tilde{h}_{1}\right)=0.3489, S\left(\tilde{h}_{2}\right)=0.4522, S\left(\tilde{h}_{3}\right)=0.2030 \\
& S\left(\tilde{h}_{4}\right)=0.2652, S\left(\tilde{h}_{5}\right)=0.2515
\end{aligned}
$$

Step 4. Rank all the candidates $A_{i}(1,2,3,4,5)$ in accordance with the scores $\mathrm{s}(\tilde{\mathrm{h}})(\mathrm{i}=1,2,3,4,5)$ of the overall interval intuitionistic trapezoidal fuzzy numbers $\tilde{h}(\mathrm{i}=1,2, \cdots, 5): \mathrm{A}_{2} \succ \mathrm{A}_{1} \succ \mathrm{A}_{4} \succ \mathrm{A}_{3} \succ \mathrm{A}_{3}$, and thus the most desirable candidate is $A_{2}$.

Based on the IITFPWG operator, then, in order to select the most desirable candidate, we can develop an approach to multiple attribute decision making problems with interval intuitionistic trapezoidal fuzzy information, which can be described as following:

\section{Step 12 . See Step 1.}

Step 22 . Aggregate all interval intuitionistic trapezoidal fuzzy numbers $\tilde{h}_{i j}(j=1,2, \cdots, n)$ using the interval intuitionistic trapezoidal fuzzy prioritized weighted geometric (IITFPWG) operator to derive the overall interval intuitionistic trapezoidal fuzzy numbers $\tilde{\mathrm{h}}_{\mathrm{i}}(\mathrm{i}=1,2, \cdots, 5)$ of the candidates $A_{i}$.

$$
\begin{aligned}
& ([0.3340,0.4544,0.5688,0.6752] ;[0.3908,0.4926] ;[0.2654,0.3464]) \\
& ([0.5448,0.6455,0.7576,0.8620] ;[0.2372,0.6390] ;[0.2067,0.3073]) \\
& ([0.1184,0.2226,0.4240,0.5254] ;[0.2885,0.4037] ;[0.1121,0.2373]) \\
& ([0.2332,0.3758,0.4808,0.5848] ;[0.3105,0.5846] ;[0.2082,0.3699]) \\
& ([0.2168,0.3178,0.4184,0.5188] ;[0.3979,0.6397] ;[0.1649,0.3201])
\end{aligned}
$$

Step 3. Calculate the scores $\tilde{h}_{i}(i=1,2, \cdots, 5)$ of the overall interval intuitionistic trapezoidal fuzzy numbers $\tilde{h}_{i}(i=1,2, \cdots, 5)$ of the candidates $A_{i}$ :

$$
\begin{aligned}
& S\left(\tilde{h}_{1}\right)=0.3489, S\left(\tilde{h}_{2}\right)=0.4522, S\left(\tilde{h}_{3}\right)=0.2030 \\
& S\left(\tilde{h}_{4}\right)=0.2652, S\left(\tilde{h}_{5}\right)=0.2515
\end{aligned}
$$

Step 4. Rank all the candidates $A_{i}(1,2,3,4,5)$ in accordance with the scores $s\left(\tilde{h_{i}}\right)(i=1,2,3,4,5)$ of the overall interval intuitionistic trapezoidal fuzzy numbers $\tilde{h}_{i}(i=1,2, \cdots, 5): A_{2} \succ A_{1} \succ A_{4} \succ A_{5} \succ A_{3}$, and thus the most desirable candidate is $A_{2}$.

In this section, we have proposed two approaches to solve the interval intuitionistic trapezoidal fuzzy multiple attribute decision making problems in which the attributes are in different priority level. From the above analysis, we can see that the main advantages of the proposed operators and approaches over the traditional interval intuitionistic trapezoidal fuzzy operators and approaches are not only due to the fact that our operators accommodate the interval intuitionistic trapezoidal fuzzy environment but also due to the consideration of the prioritization among the attributes, which makes it more feasible and practical.

\section{Conclusion}

In this paper, we investigate the interval intuitionistic trapezoidal fuzzy multiple attribute decision making (MADM) problem in which the attributes are in different priority level. Then, motivated by the ideal of prioritized aggregation operators[33], we have developed some prioritized aggregation operators for aggregating interval intuitionistic trapezoidal fuzzy information: interval intuitionistic trapezoidal fuzzy prioritized weighted average (IITFPWA) operator and interval intuitionistic trapezoidal fuzzy numbers prioritized weighted geometric (ITFPWG) operator. The prominent characteristic of these proposed operators is that they take into account prioritization among the attributes. Then, we have utilized these operators to develop some approaches to solve the interval intuitionistic trapezoidal fuzzy multiple attribute decision making problems in which the attributes are in different priority level. Finally, a practical example about talent introduction is given to verify the developed approach and to 
demonstrate its practicality and effectiveness.

\section{References}

[1] Atanassov, K. (1986). Intuitionistic fuzzy sets, Fuzzy Sets and Systems, 20, 87-96.

[2] Atanassov, K. (1989). More on intuitionistic fuzzy sets, Fuzzy Sets and Systems 33. 37-46.

[3] Atanassov, K. (2000). Two theorems for intuitionistic fuzzy sets, Fuzzy Sets and Systems 110, 267-269.

[4] Zadeh, L. A. (1965). Fuzzy sets, Information and Control 8. 338-356.

[5] Atanassov, K., Gargov, G. (1989). Interval-valued intuitionistic fuzzy sets, Fuzzy Sets and Systems 31, 343-349.

[6] Atanassov, K. (1994). Operators over interval-valued intuitionistic fuzzy sets, Fuzzy Sets and Systems 64 (2) 159-174.

[7] Chen, T.Y., Wang, H. P., Lu, Y. Y. (2011). A multicriteria group decision-making approach based on interval-valued intuitionistic fuzzy sets: A comparative perspective. Expert Systems with Applications, 38, 7647-7658.

[8] Devi, K. (2011). Extension of VIKOR method in intuitionistic fuzzy environment for robot selection. Expert Systems with Applications, 38, 14163-14168.

[9] Li, D.F. (2010). Linear programming method for MADM with interval-valued intuitionistic fuzzy sets. Expert Systems with Applications, 37, 5939-5945.

[10] Li, D.F. (2010). Multiattribute decision making method based on generalized OWA operators with intuitionistic fuzzy sets. Expert Systems with Applications, 37, 8673-8678.

[11] Guiwu Wei., Xiaofei Zhao. (2012). Some dependent aggregation operators with 2-tuple linguistic information and their application to multiple attribute group decision making, Expert Systems with Applications, 39, 5881-5886.

[12] Wei, G. W., Zhao, X. F. (2012). Some induced correlated aggregating operators with intuitionistic fuzzy information and their application to multiple attribute group decision making, Expert Systems with Applications, 39 (2), 2026-2034.

[13] Wei, G.W., Wang, H. J., Lin, R., Zhao, X. F. (2011). Grey relational analysis method For intuitionistic fuzzy multiple attribute decision making with preference information on alternatives, International Journal of Computational Intelligence Systems, 4 (2) 164-173.

[14] Wei, G.W. (2010). GRA method for multiple attribute decision making with incomplete weight information in intuitionistic fuzzy setting. Knowledge-Based Systems, 23, 243-247.

[15] Wei, G.W. (2010). Some induced geometric aggregation operators with intuitionistic fuzzy information and their application to group decision making. 10, 423-431.

[16]Wei, G.W. Gray relational analysis method for intuitionistic fuzzy multiple attribute decision making. Expert Systems with Applications, 38, 11671-11677.

[17] Wei, G.W., X.F. Zhao. (2011). Minimum deviation models for multiple attribute decision making in intuitionistic fuzzy setting, International Journal of Computational Intelligence Systems, 4 (2) 174-183.

[18] Xu, Z.S.(2007). Intuitionistic fuzzy aggregation operators. IEEE Transactions on Fuzzy Systems, 15. 1179-1187.

[19] Xu, Z.S. (2010). Choquet integrals of weighted intuitionistic fuzzy information. Information Sciences, 180 . 726-736.

[20] Xu, Z.S. (2011). Approaches to multiple attribute group decision making based on intuitionistic fuzzy power aggregation operators. Knowledge-Based Systems, 24. 749-760.

[21] Xu, Z.S., Xia, M. M. (2011). Induced generalized intuitionistic fuzzy operators. Knowledge-Based Systems, 24, 197-209.

[22] Yue, Z.L. (2011). An approach to aggregating interval numbers into interval-valued intuitionistic fuzzy information for group decision making. Expert Systems with Applications,38. 6333-6338.

[23] Chen, T.Y. (2010). An outcome-oriented approach to multicriteria decision analysis with intuitionistic fuzzy optimistic/pessimistic operators. Expert Systems with Applications, 37, 7762-7774.

[24] Ye, J. (2010). Fuzzy decision-making method based on the weighted correlation coefficient under intuitionistic fuzzy environment. European Journal of Operational Research, 205, 202-204.

\begin{tabular}{llllll}
\hline 12 & Journal of Intelligent Computing Volume & 9 & Number & 3 & September \\
\hline
\end{tabular}


[25] Ye, J. (2011)Expected value method for intuitionistic trapezoidal fuzzy multicriteria decision-making problems. Expert Systems with Applications , 38, 11730-11734.

[26] Ye, J. Fuzzy cross entropy of interval-valued intuitionistic fuzzy sets and its optimal decision-making method based on the weights of alternatives. Expert Systems with Applications 38. 6179-6183.

[27] Shu, M H, Cheng, C H., Chang, J R. (2006). Using intuitionistic fuzzy sets for fault-tree analysis on printed circuit board assembly, Microelectronics Reliability, 46. 2139-2148.

[28] Wang Jianqiang. (2008). Overview on fuzzy multi-criteria decision-making approach,. Control and decision, 23 (6) 601-606.

[29] Wang Jianqiang., Zhang Zhong. (2008). Programming method of multi-criteria decision-making based on intuitionistic fuzzy number with incomplete certain information,. Control and decision, 23(10) 1145-1148.

[30] Wang Jianqiang., Zhang Zhong. (2009). multi-criteria decision-making method with incomplete certain information based on intuitionistic fuzzy number. Control and decision, 24 (2) 226-230.

[31] Wan Shuping. (2011). Multi-attribute Decision Making Method based on Interval Intuitionistic Trapezoidal Fuzzy Number. Control and decision, 26(6) 857-860.

[32] Wei, G. W. (2012). An approach to multiple attribute group decision making with interval intuitionistic trapezoidal fuzzy information, Technological and Economic Development of Economy, 2012. In press

[33] Yager, R. R. (2008). Prioritized aggregation operators, International Journal of Approximate Reasoning 48, 263-274.

[34] Yager, R.R. (2009). Prioritized OWA aggregation, Fuzzy Optimization Decision Making, 8, 245-262.

[35] Yu, D. et al. (2011). Interval-valued intuitionistic fuzzy prioritized operators and their application in group decision making, Knowledge-based Systems. 